
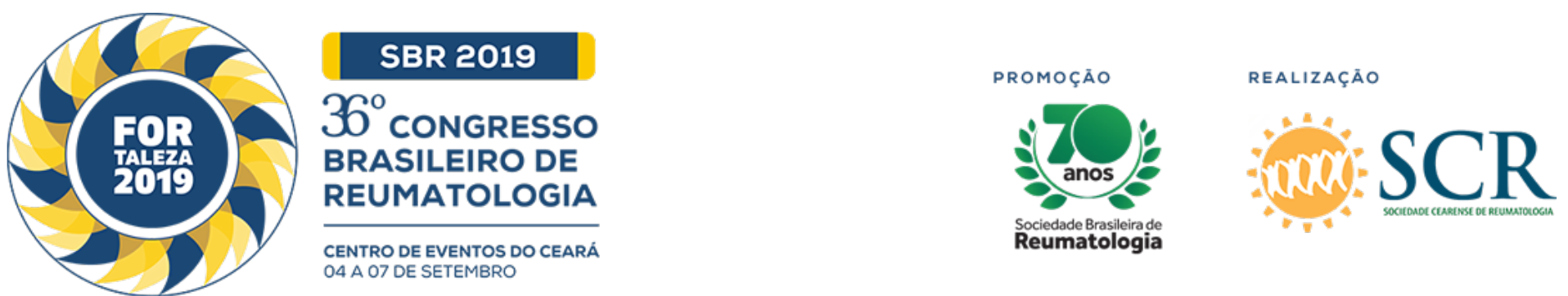

\title{
CLINICAL AND LABORATORIAL MANIFESTATIONS OF SYSTEMIC LUPUS ERYTHEMATOSUS IN CHILDREN AND ADOLESCENTS
}

Rogério do Prado (Faculdade de Medicina do ABC, Santo André, SP, Brasil), Thais Akemi Yoshimoto (Faculdade de Medicina do ABC, Santo André, SP, Brasil), Aline Rodrigues Loreto (Faculdade de Medicina do ABC, Santo André, SP, Brasil), Beatriz Jeronymo Pardi (Faculdade de Medicina do ABC, Santo André, SP, Brasil), Roseli O S Sarni (Faculdade de Medicina do ABC, Santo André, SP, Brasil)

\section{BACKGROUND}

Systemic lupus erythematosus (SLE) is a chronic, multisystemic inflammatory disease of unknown cause and autoimmune in nature. It is characterized by tissue damage mediated by autoantibodies, and can affect skin, joints, kidneys, central nervous system, cardiovascular system, serous, hematological system and immune system. The diagnosis is made according to the SLICC SLE classification criteria (Systemic Lupus International Collaborating Clinics, 2012). There is a shortage of studies on juvenile SLE in the world and Brazilian literature. This pathology has peculiar characteristics in this age group that should be better defined.

\section{MATERIALS AND METHODS}

A cross - sectional retrospective study was carried out by means of a review of all medical records of patients aged 0 to 21 years who met the SLE - SLICC diagnostic criteria from January 2014 to July 2016. Data from the initial clinical and laboratory presentation were collected and analyzed of the SLE, from the beginning of the clinical picture to the diagnosis, such as: sex, age of diagnosis, clinical and laboratory manifestations according to the SLICC criteria.

\section{RESULTS}

Fourteen patients with SLE criteria were identified. The age at onset of the disease was 6 to 16 years, the mean was 138.85 months. Only 3 patients $(21.4 \%)$ were less than 10 years old at the time of diagnosis. Regarding gender, $85.7 \%$ are female, the proportion of women to men was $6: 1$. The initial manifestation of the disease in males occurred in the younger patients in the 6 and 9 years old group, and the presentation in the form of renal impairment and pleural effusion, respectively. None of the boys presented the cutaneous form. The initial manifestations will be shown in Table 1 and the immunological manifestations will be presented in Table 2

\section{CONCLUSION}

In our study we found results that, in general, agree with the data of the world literature. However, it is clear that further studies on childhood SLE are needed to provide more consistent data on the clinical and laboratory presentation of SLE. 